\title{
Melittopalynological Study of Squeezed Summer Honeys Collected from Forest Area of Sindewahi Tahsil of Chandrapur District, (Maharashtra state)
}

\author{
Laxmikant Borkar ${ }^{1}$, Devendra Mate ${ }^{2}$ \\ ${ }^{1}$ Department of Botany, S. S. Jaiswal Arts, Comm and Science College, Arjuni Morgaon. Dist.Gondia-441701 \\ ${ }^{2}$ Department of Botany, Nutan Adrsh Arts, Comm, and smt M. H. Wegad Science College, Umrer. Dist. Nagpur-441203
}

\begin{abstract}
The paper incorporates a qualitative and quantitative analysis of pollen contents in Six squeezed honey samples of Apisdorsata hives collected from forest area of Sindewahi tahsil of Chandrapur district. Terminaliasp. represents the predominant pollen type in five samples ranged from (47.13\% to $75.47 \%)$ are designated as Terminaliahoney. The other significant pollen types recorded include Pongamiapinnata, Delonixregia, Mangiferaindica, Sapindusemarginatus, Syzygiumcumini, Azadirachtaindica. The pollen counts ranged from 161, 000/g to 456, 000/g. The data reflects the floral situation of the place where particular honey was produced and the identification of geographical origin based on the presence of a combination of pollen types of that particular area.
\end{abstract}

Keywords: Pollen, Honey, Apisdorsata, Forest area, Sindewahi tahsil

\section{Introduction}

Melittopalynology is an applied branch of palynology dealing with the study of pollen grains in honey samples and its application in Apiculture. Plant produces nectar and pollen both of which are avidly sought after by the bees to provide nutrition to the colony. Melittopalynology is concerned with the identification of pollen in honeys. Evaluation of plants for their utility as sources of bee forage provides the information needed to assess the potential for bee keeping in an area.

Melittopalynological studies are thus helpful in bee management and in promoting the beekeeping development. Laboratory studies using Melittopaloynological methods have been made to evaluate sources of pollen and nectar for honey bees in different parts of the country namely Maharashtra (Bhusari et al., 2005; Phadke, 1962; Kumar and Jagtap, 1988; Kalkar and Shende, 2009, 2010; Mate, 2013), Andra Pradesh (Ramanujam and Khatija, 1991, Kalpana and Ramanujam, 1991, Moses, 1987, Karnataka (Yoganarasimhan, 1982; Agashe and Ranjaswami, 1997; Sheshagri, 1985; Bhargava et al., 2009), Lucknow (Suryanarayana, 1976) and Indian honeys (Sen and Banarjee, 1956; Nair, 1964; Seethalakshmi, 1993). Present investigation incorporates a quanlitative and quantitative pollen analysis of Six honey sample from forest area of Sindewahi tahsil of Chandrapur District. In order to identify the chief bee foraging plants recognize the uni and multifloral honeys and identify areas suitable for beekeeping industry in this area. It is further investigated that a study of this nature would also highlight the geographical source of the honey samples.

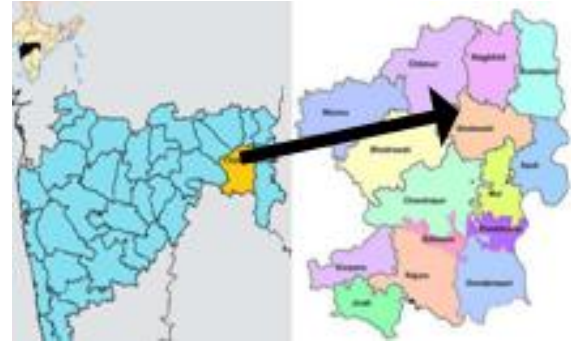

Figure 1: Map of Maharashtra Showing Chandrapur District \& Sindewahi Tahsil

\section{Literature Survey}

"Melittopalynology, one of the branches of palynology finds a very significant application in the field of apiculture. It deals with a critical microscopic study involving qualitative and quantitative analysis of the myriad pollen contents of honeys and pollen loads of bees and bee hives at diverse floristic and geographical regions, (Ramanujam, 1991). Palynological studies also help to detect pollen from poisonous plants which are often toxic not only to bees but also to human beings (Deodikar and Thakar, 1954; Deodikaret.al., 1958; Deodikar, 1960). It is rather surprising that most of the poisonous plants in the hilly regions of Mahabaleshwar, flower during January-February which represents the dearth period with acute shortage of nectar and pollen from normal sources. Bees, therefore visit these poisonous plants due to sheer starvation (Chaubal and Deodikar, 1963)

\section{Materials and Methods}

Six honey samples viz., CHN-SIN-Naw, CHN-SIN-Gad, CHN-SIN-Was, CHN-SIN-Shi, CHN-SIN-Kag, CHN-SINDel were collected during the period 03 May 2012 to 12 Jun 2013 from Nawargaon, Gadbori, Wasera, Shirkhada, Kargata, Delanwahi respectively. All the samples represent squeezed honey collected from the natural Apisdorsata hives (Map). 


\section{International Journal of Science and Research (IJSR) \\ ISSN (Online): 2319-7064 \\ Index Copernicus Value (2013): 6.14 | Impact Factor (2015): 6.391}

The squeezing (pressing) of the honey combs was carried out under personal supervision and only honey bearing portion of the comb was used for this purpose. $1 \mathrm{ml}$ of the honey sample was dissolved in $10 \mathrm{ml}$ of distilled water \& centrifuged. The sediment obtained was treated with $5 \mathrm{ml}$ glacial acetic acid. The acetic acid was decanted and the material was subjected to acetolysis (Erdtman, 1960) for analysing the pollen content in honeys qualitatively \& quantitatively, three pollen slides were prepared for each sample. The recorded pollen types were identified with the help of reference slides collection \& relevant literature for quantification of pollen types recorded, a total of 300 pollen grains were counted at random from the three palynoslides prepared for each samples. Based on their frequencies, the pollen types encountered were placed under the pollen frequency classes recommended by the international commission for bee Botany Louveaux et al;(1978) viz., predominant pollen type $(>45 \%)$, secondary pollen type (16$45 \%$ ), important minor pollen types (3-15\%), and minor pollen types $(<3 \%)$.Non-melliferous (anemophilous) pollen types were excluded while determine the frequencies of melliferous pollen types (International Commission for Bee Botany Louveaux et al. ;1978). The absolute pollen count of each sample was determined in accordance with the method recommended by Suryanarayana et al. (1981). Unacetolysed samples of honey were examined for the study of honeydew elements (fungal spores, hyphal threads and algal filaments).

\section{Results and Discussion}

Of the 6 honey samples collected from Sindewahi tahsil, Terminaliasp. ranged from $(47.13 \%$ to $75.47 \%)$ represented the predominant pollen type in five sample (CHN-SINNaw), (CHN-SIN-Gad), (CHN-SIN-Was), (CHN-SIN-Shi), (CHN-SIN-Del)i.e. Unifloral while 1 is multifloral $(\mathrm{CHN}-$ SIN-Kag). The other significant pollen types recorded includes (secondary to minor pollen) Terminaliasp., Pongamiapinnata, Delonixregia, Syzygiumcumini, Mangiferaindica, Sapindusemarginatus, Pisidiumguajava, Asterecanthalongifolia, Rungiarepens, Azadirachtaindica .

Table 1: Pollen frequency class \& frequencies $(\%)$ in Apisdorsata summer honeys

\begin{tabular}{|c|c|c|c|c|c|}
\hline Sample No. & $\begin{array}{c}\text { Date of } \\
\text { Collection }\end{array}$ & $\begin{array}{l}\text { Type of } \\
\text { Honey }\end{array}$ & $\begin{array}{l}\text { Absolute } \\
\text { pollen counts } \\
(\mathrm{APC}) / \mathrm{g}\end{array}$ & $\mathrm{HDE} / \mathrm{P}$ & Pollen Type \\
\hline $\begin{array}{c}\text { CHN-SIN- } \\
\text { Naw }\end{array}$ & 03-05-2012 & Unifloral & $205,000 / \mathrm{g}$ & 0.01 & $\begin{array}{c}\text { P - Terminalia sp. }(47.13) \\
\text { S - Pongamiapinnata(17.14) } \\
\text { Delonixregia(16.81) } \\
\text { I - Asteracanthalongifolia }(4.10) \\
\text { Leucaenaleucocephala }(4.06) \\
\text { Cucurbitaceae type }(3.02) \\
M-S y(2.08), E u(2.04), \text { Bi }(1.04), \text { Br }(1.02), \text { All }(0.98), \text { Sap }(0.68) \\
N M P-\text { Sorghum vulgare }(0.18) \\
\end{array}$ \\
\hline $\begin{array}{c}\text { CHN-SIN- } \\
\text { Gad }\end{array}$ & 08-05-2012 & Unifloral & $19,000 / \mathrm{g}$ & 0.03 & $\begin{array}{c}\text { P - Terminalia sp.(61.35) } \\
\text { S - Nil } \\
\text { I - Syzygiumcumuni(13.83) } \\
\text { Psidiumguajava(7.06) } \\
\text { Mangiferaindica, Pongamiapinnata(each 4.02) } \\
\text { Delonixregia(3.92) } \\
\mathrm{M}-\text { Ci(1.82), Son(1.04), Car, Cas }(\text { each } 0.86), \mathrm{He}(0.82) \\
\mathrm{NMP}-\text { Sorghum vulgare }(0.72) \\
\end{array}$ \\
\hline $\begin{array}{c}\text { CHN-SIN- } \\
\text { Was }\end{array}$ & 09-05-2013 & Unifloral & $187,000 / \mathrm{g}$ & 0.01 & $\begin{array}{c}\mathrm{P}-\text { Terminalia sp. }(66.67) \\
\mathrm{S}-\text { Mangiferaindica(20.58) } \\
\mathrm{I} \text { - Azadirachtaindica }(4.47) \\
\text { Pongamiapinnata }(3.22) \\
\mathrm{M}-\text { Ast.l(1.97), Bo }(1.56), \\
\text { Cell }(1.04), \\
N M P-\text { Sorghum vulgare }(0.72) \\
\end{array}$ \\
\hline $\begin{array}{c}\text { CHN-SIN- } \\
\text { Shir }\end{array}$ & $12-05-2013$ & Unifloral & $456,000 / \mathrm{g}$ & 0.01 & $\begin{array}{c}\mathrm{P}-\text { Terminalia sp.(75.47) } \\
\mathrm{S}-\text { Sapindusemarginatus(17.43) } \\
\mathrm{I}-\mathrm{Nil} \\
\mathrm{M}-\text { Ast.l(2.06), Bo, De(each 1.06), Ma(0.68), Caps(0.21), Car, } \\
\text { May(each O.33), Ju(0.86), } \\
\mathrm{NMP}-\mathrm{Nil}\end{array}$ \\
\hline $\begin{array}{c}\text { CHN-SIN- } \\
\text { Kag }\end{array}$ & 27-05-2013 & Multifloral & $161,000 / \mathrm{g}$ & 0.02 & $\begin{array}{c}\text { P - Nil } \\
\text { S - Terminalia sp.(31.75) } \\
\text { Mangiferaindica }(20.16) \\
\text { Delonixregia(17.06) } \\
\text { I - Pongamiapinnata (14.15) } \\
\text { Syzygiumcumuni (6.42) } \\
\text { M- Cel(2.08), Ast.l, Ps(each 2.04), Bo(1.86), Car(1.06), May (1.04), } \\
\text { Caps }(0.43) \\
\text { NMP - Typhaangustata(11.58) } \\
\text { Sorghum vulgare(4.16) }\end{array}$ \\
\hline
\end{tabular}


International Journal of Science and Research (IJSR)

ISSN (Online): 2319-7064

Index Copernicus Value (2013): 6.14 | Impact Factor (2015): 6.391

\begin{tabular}{|c|c|c|c|c|c|}
\hline $\begin{array}{c}\text { CHN-SIN- } \\
\text { Del }\end{array}$ & $12-06-2013$ & Unifloral & $209,000 / \mathrm{g}$ & 0.02 & P - Terminalia sp. (50.26) \\
& & & & & S - Syzygiumcumuni (34.91) \\
& & & & I-Asteracanthalongifolia (4.11) \\
& & & & Rungiarepens(3.84) \\
& & & & M -De(2.84), Tri(1.84), Son(1.64), Pr(1.06) \\
NMP-Sorghum vulgare(0.72)
\end{tabular}

Table 2: Showing pollen morphology of Melliferous taxa

\begin{tabular}{|c|c|c|c|c|}
\hline \begin{tabular}{|l|} 
Sr. \\
No.
\end{tabular} & Pollen types & Pollen Size, Shape and Symmetry & Aperture pattern & $\begin{array}{c}\text { Pollen wall (Sporoderm) Structure and } \\
\text { sculpture }\end{array}$ \\
\hline 01 & Allium cepa Linn. & $\begin{array}{c}14-28 \times 32-48 \mu \mathrm{m}, \text { ellipsoidal } \\
\text { Bilaterally symmetrical }\end{array}$ & Monosulcate, sulcus tenuimarginate & $\begin{array}{c}\text { Exine } 1.5 \mu \mathrm{m} \text { thick, subtectate, surface } \\
\text { faintly reticulate }\end{array}$ \\
\hline 02 & $\begin{array}{l}\text { Asteracanthalongifolia } \\
\text { (Linn.) Nees. }\end{array}$ & $\begin{array}{c}56-59 \mu \mathrm{m}, \text { Amb spheroidal or } \\
\text { quadrangular; } 50-55 \times 52-59 \mu \mathrm{m} \text {, } \\
\text { oblate spheroidal; Radially } \\
\text { symmetrical }\end{array}$ & $\begin{array}{c}\text { Tetracolporate, colpi long, ends } \\
\text { tapering, tips acute, colpi alternating } \\
\text { with } 4 \text { streak like pseudocolpi, ora } \\
\text { more or less circular. }\end{array}$ & $\begin{array}{c}\text { Exine } 3.3 \mu \mathrm{m} \text { thick, subtectate, } \text { surface } \\
\text { reticulate, homobrochate, lumina } \\
\text { polygonal and psilate. }\end{array}$ \\
\hline 03 & AzadirachtaindicaA.juss & $\begin{array}{c}50-54 \mu \mathrm{m}, \text { Ambsquarish, sides } \\
\text { convex; } 47-5438-47 \mu \mathrm{m}, \\
\text { subprolate, poles smoothly } \\
\text { rounded; Radially symmetrical }\end{array}$ & $\begin{array}{l}\text { Tetracolporate, colpi long, ends } \\
\text { tapering, tips acute, oralalongate }\end{array}$ & $\begin{array}{c}\text { Exine } 3 \mu \mathrm{m} \text { thick, tectate, surface } \\
\text { psilate to locally granular }\end{array}$ \\
\hline 04 & Brassica sp.(Linn) Koch & \begin{tabular}{|c|}
$30-33 \mu \mathrm{m}$, Amb rounded \\
triangular to almost spheroidal; \\
$27-31 \times 24-27 \mu \mathrm{m}$, prolate \\
spheroidal; radially symmetrical \\
\end{tabular} & $\begin{array}{l}\text { Tricolplate , colpal ends tapering, tips } \\
\text { acute }\end{array}$ & $\begin{array}{c}\text { Exine } 2.5 \mu \mathrm{m} \text { thick, sub tectate, surface } \\
\text { reticulate, heterobrochate, meshes } \\
\text { narrow at mesocolpial regions giving a } \\
\text { striate look, lumina polygonal. }\end{array}$ \\
\hline 05 & Bombaxceiba Linn & $\begin{array}{c}51 \mu \mathrm{m}(49.5 \times 52.5) \mu \mathrm{m} \text {, peroblate, } \\
\text { isopolar, Radially symmetrical }\end{array}$ & $\begin{array}{c}\text { Tricolprate, col. length } 12(10.5-13.5) \\
\mu \mathrm{m}\end{array}$ & $\begin{array}{c}\text { Exine thick } 3 \mu \mathrm{m}, \text { coarsely reticulate, } \\
\text { mesh } 4.1 \mu \mathrm{m}(3-4.5 \mu \mathrm{m}) \text { in the major } \\
\text { part except at the angles showing } \\
\text { medium reticulations } 1-8 \mu \mathrm{m}(1.5-3 \\
\mu \mathrm{m}), \text { greater number of baculae are } \\
\text { found in the lumen. } \\
\text { Murisimplibaculate, faint LO pattern. }\end{array}$ \\
\hline 06 & Bidenapilosa Linn. & $\begin{array}{c}25-29 \mu \mathrm{m} \text { Amb spheroidal; } 23- \\
25 \times 27-30 \mu \mathrm{m}, \text { sub-oblate; } \\
\text { Radially symmetrical } \\
\end{array}$ & $\begin{array}{c}\text { Tricolporate, colpi long, ends tapering, } \\
\text { tips acute, oralalongate }\end{array}$ & $\begin{array}{c}\text { Exine } 1.5 \mu \mathrm{m} \text { thick, tectate, surface } \\
\text { echinate, spines } 6.8 \mu \mathrm{m} \text { long, base } 2 \mu \mathrm{m} \\
\text { broad }\end{array}$ \\
\hline 07 & CareyaarboreaRoxb. & $\begin{array}{l}52.1 \times 40.1 \mu \mathrm{m}(48-54 \times 37.5- \\
43.5) \mu \mathrm{m}, \text { subprolate, isopolar, } \\
\text { radially symmetrical }\end{array}$ & $\begin{array}{c}\text { Hexacolpate, syncolpate with } \\
\text { crassimarginatecolpi, col. Length } 43.5 \\
(42-46.5) \mu \mathrm{m}\end{array}$ & \begin{tabular}{|} 
Exinethick, $3 \mu \mathrm{m}$, undulating, \\
considerable thick at the poles sexine- \\
nexine not differentiated medium \\
reticulate, more coarse at the poles. \\
Mesh $1.5-3 \mu \mathrm{m}$, clear LO pattern \\
\end{tabular} \\
\hline 08 & Citrus sp. & $\begin{array}{l}27-29 \mu \mathrm{m}, \text { Ambsquarish, } 26-30 \\
\times 25-27 \mu \mathrm{m}, \text { prolate spheroidal } \\
\text { radially symmetrical }\end{array}$ & $\begin{array}{c}\text { Tetracolporate, colpi linear, tips acute, } \\
\text { oralalongate }\end{array}$ & $\begin{array}{c}\text { Exine } 2 \mu \mathrm{m} \text { thick subtectate, surface } \\
\text { Reticulate. Heterobrochate, meshes } \\
\text { smaller near the apertural regions and } \\
\text { larger elsewhere, luminahexa to } \\
\text { pentagonal or irregular, psilate, } \\
\text { murisimpli to locally duplibaculate }\end{array}$ \\
\hline 09 & $\begin{array}{l}\text { CaseariaellipticaWilld } \\
\end{array}$ & \begin{tabular}{|c|}
$29-37 \mu \mathrm{m}$, Amb spheroidal ; $28-36$ \\
$\times 27-33 \mu \mathrm{m}$ subprolate radially \\
symmetrical
\end{tabular} & $\begin{array}{c}\text { Tricolporate , colpi with tapering ends, } \\
\text { ora } \\
\text { lalongate }\end{array}$ & $\begin{array}{c}\text { Exine } 1.5 \mu \mathrm{m} \text { thick, tectate, surface } \\
\text { psilate }\end{array}$ \\
\hline 10 & Capsicum annuum Linn. & $\begin{array}{c}29-34 \mu \mathrm{m}, \text { Amb spheroidal; } 29- \\
\begin{array}{c}35 \times 26-30 \mu \mathrm{m}, \text { subprolate; } \\
\text { radially symmetrical }\end{array}\end{array}$ & $\begin{array}{c}\text { Tricolporate, colpi constricted at oral } \\
\text { region, ends tapering, tips acute, ora } \\
\text { prominently lalongate }\end{array}$ & $\begin{array}{l}\text { Exine } 1.5 \mu \mathrm{m} \text { thick, tectate, surface } \\
\text { faintly granular to almost psilate }\end{array}$ \\
\hline 11 & Celosia argentea Linn & $\begin{array}{l}\text { 30-35 } \mu \mathrm{m} \text { spheroidal radially } \\
\text { symmetrical }\end{array}$ & $\begin{array}{c}\text { Pantoporate, pore No. } 15-20 \text {, circular. } \\
\text { Diam; } 4-5 \mu \mathrm{m} \text {, pore membrance } \\
\text { flecked with granules, interporal } \\
\text { distance } 8-11 \mu \mathrm{m}\end{array}$ & $\begin{array}{c}\text { Exine } 2 \mu \mathrm{m} \text { thick, tectate, interporal } \\
\text { space coarsely granular }\end{array}$ \\
\hline 12 & Clerodendrum sp. & $\begin{array}{c}43-48 \mu \mathrm{m}, \text { Amb spheroidal, } 41-44 \\
\times 37-40 \mu \mathrm{m}, \text { prolate spheroidal } \\
\text { Radially symmetrical }\end{array}$ & $\begin{array}{l}\text { Tricolporate, colpi fairly long, tips } \\
\text { acute, colpal margins broken }\end{array}$ & $\begin{array}{c}\text { Exine } 1.5 \mu \mathrm{m} \text { thick (excluding } \\
\text { spinules), tectate surface spinulate, } \\
\text { spinules } 0.6-1 \mu \mathrm{m} \text { long, interspinular } \\
\text { space finely granular }\end{array}$ \\
\hline 13 & Cucurbitaceae type & $\begin{array}{c}\text { 50-63 } \mu \mathrm{m} \text {, subprolate, isopolar, } \\
\text { Radially symmetrical }\end{array}$ & $\begin{array}{l}\text { Tricolporate, col. Length } 48.7 \text { (48- } \\
\text { 49.5) } \mu \mathrm{m}\end{array}$ & $\begin{array}{c}\text { Exine } 4.5 \mu \mathrm{m} \text { thick, sexine - nexine not } \\
\text { discernible, rather coarsely reticulate, } \\
\text { mesh } 3.4\left(\begin{array}{c}3-4.5) \mu \mathrm{m}, \text { baculae distinct } \\
\text { clear LO pattern }\end{array}\right.\end{array}$ \\
\hline 14 & $\begin{array}{l}\text { Delonixregia (Boj. ex. } \\
\text { Hoof.) Ref. }\end{array}$ & $\begin{array}{c}59.62 \mu \mathrm{m}, \text { Amb more or less } \\
\text { spheroidal to subtriangular; } 53- \\
56 \times 57-60 \mu \mathrm{m} \text {, oblate to } \\
\text { suboblate; Radially symmetrical }\end{array}$ & $\begin{array}{l}\text { Tricolporate, colpi long with blunt } \\
\text { ends, ora faint, more or less rounded }\end{array}$ & \begin{tabular}{|c|} 
Exine $5.2 \mu \mathrm{m}$ thick, subtectate, surface \\
coarsely reticulate. Heterobrochate, \\
meshes smaller near the apertural \\
regions \& larger elsewhere, lumina \\
poly to hexagonal with a number of
\end{tabular} \\
\hline
\end{tabular}

Volume 5 Issue 6, June 2016 www.ijsr.net

Licensed Under Creative Commons Attribution CC BY 


\section{International Journal of Science and Research (IJSR) \\ ISSN (Online): 2319-7064}

Index Copernicus Value (2013): 6.14 | Impact Factor (2015): 6.391

\begin{tabular}{|c|c|c|c|c|}
\hline & & & & $\begin{array}{l}\text { free bacules, muri thick, sinuous, } \\
\text { simpli to locally duplibaculate }\end{array}$ \\
\hline 15 & Eucalyptus sp. & $\begin{array}{c}23-27 \mu \mathrm{m}, \text { Amb triangular, sides } \\
\text { concave to almost flat; } 15-18 \times \\
25-29 \mu \mathrm{m}, \text { oblate, Radially } \\
\text { symmetrical }\end{array}$ & $\begin{array}{c}\text { Tricolporate, syncolpate, } \\
\text { parasyncolpate, oralalongate, margins } \\
\text { incrasste }\end{array}$ & $\begin{array}{c}\text { Exine } 1.5 \mu \mathrm{m} \text { thick, much thickened at } \\
\text { angles, tectate, surface psilate }\end{array}$ \\
\hline 16 & Helianthus annuus Linn. & $\begin{array}{c}40-44 \mu \mathrm{m}, \text { Amb spheroidal, } 37- \\
39 \times 40-42 \mu \mathrm{m}, \text { oblate spheroidal; } \\
\text { Radially symmetrical }\end{array}$ & $\begin{array}{c}\text { Tricolporate , colpal ends tapering, } \\
\text { oralalongate }\end{array}$ & $\begin{array}{c}\text { Exine } 3 \mu \mathrm{m} \text { thick (without spines), } \\
\text { ectate, surface densely echinate, spines } \\
7-8 \mu \mathrm{m} \text { long, base } 2.4 \mu \mathrm{m} \text { wide, tip } \\
\text { pointed. }\end{array}$ \\
\hline $17 \mathrm{~J}$ & Justiciaprocumbens Linn. & $\begin{array}{l}24-28 \times 16-18 \mu \mathrm{m}, \text { onlong; } \\
\text { Bilaterally symmetrical }\end{array}$ & $\begin{array}{l}\text { Dicolporate, colpi faint, narrow, streak } \\
\text { like, oralalongate }\end{array}$ & $\begin{array}{l}\text { Exine } 1 \mu \mathrm{m} \text { thick at poles, } 2.5 \mu \mathrm{m} \text { thick } \\
\text { at equator, tectum undulating, circular } \\
\text { to irregular are poles }(2-4 \mu \mathrm{m}) \text { aligned } \\
\text { linearly are seen on either side of the } \\
\text { colpi, rest of the wall finely reticulate }\end{array}$ \\
\hline 18 & $\begin{array}{l}\text { Leucaenaleucocephala } \\
\text { (Lam.) de Wit }\end{array}$ & $\begin{array}{c}52-59 \mu \mathrm{m}, \text { Amb spheroidal : } 47- \\
49 \times 51-58 \mu \mathrm{m} \text {, sub oblate: } \\
\text { Radially symmetical }\end{array}$ & $\begin{array}{c}\text { Tricolporatecolpilong, tips acute, } \\
\text { oralalongate }\end{array}$ & $\begin{array}{l}\text { Exine } 4 \mu \mathrm{m} \text { thick, subtectate surface } \\
\text { microreticulate, homobrochate }\end{array}$ \\
\hline 19 & Mangiferaindica Linn. & $\begin{array}{c}27-31 \mu \mathrm{m}, \text { Ambsubtriangular; } 29- \\
32 \times 26-28 \mu \mathrm{m}, \text { subprolate; } \\
\text { Radially symmetrical }\end{array}$ & $\begin{array}{c}\text { Tricolporatecolpi long, tips acute ora } \\
\text { prominently lanlongate }\end{array}$ & $\begin{array}{c}\text { Exine } 2.5 \mu \mathrm{m} \text { thick, subtectate, surface } \\
\text { striatoreticulae, striations more or less } \\
\text { parallel in equatorial view, lumen } \\
\text { generally elongated in polar direction, } \\
\text { murisimplibaculate }\end{array}$ \\
\hline 20 & $\begin{array}{l}\text { Maytenusemarginata } \\
\text { Wild. }\end{array}$ & \begin{tabular}{|c|} 
Oblate, $45-49 \mu \mathrm{m}, \mathrm{Amb}$, rounded \\
triangular to almost spheroidal, \\
isopalar, Radially symmetrical
\end{tabular} & \begin{tabular}{|} 
Tricoloporate, colpi length $9.4 \mu \mathrm{m},(9-$ \\
$10.5) \mu \mathrm{m}$, oralalongate
\end{tabular} & $\begin{array}{c}\text { Exine thick } 3 \mu \mathrm{m} \text {, sexine thicker than } \\
\text { nexine, reticulate size of mesh } 2.4(1.5- \\
\text { 3) } \mu \mathrm{m} \text {, distinct LO pattern. }\end{array}$ \\
\hline 21 & \begin{tabular}{|l|}
$\begin{array}{l}\text { Pongamiapinnata (Linn) } \\
\text { Pierre. }\end{array}$ \\
\end{tabular} & $\begin{array}{c}29-31 \mu \mathrm{m}, \text { Ambsubtriangular: } 27- \\
31 \times 25-28 \mu \mathrm{m}, \text { subprolate; } \\
\text { Radially symmetrical }\end{array}$ & $\begin{array}{c}\text { Tricolporate, colpi linear to narrowly } \\
\text { elliptic tips acute, oralalongate }\end{array}$ & $\begin{array}{c}\text { Exine } 1.5 \mu \mathrm{m} \text { thick, subtectate, surface } \\
\text { granular to locally faintly } \\
\text { microrecticulate }\end{array}$ \\
\hline 22 & $\begin{array}{c}\text { Prosopisjuliflora (Sw.) } \\
\text { DC }\end{array}$ & $\begin{array}{c}36-39 \mu \mathrm{m}, \text { Amb rounded } \\
\text { triangular; } 38-42 \times 30-35 \mu \mathrm{m}, \\
\text { prolate to subprolate; Radially } \\
\text { symmetrical }\end{array}$ & $\begin{array}{c}\text { Tricolllporate, occasionally syncolpate, } \\
\text { colpi tapering towards poles, tips acute, } \\
\text { oralalongate }\end{array}$ & $\begin{array}{c}\text { Exine } 3.2 \mu \mathrm{m} \text { thick, tectate surface } \\
\text { faintly reticulate }\end{array}$ \\
\hline 23 & \begin{tabular}{l|} 
Pisidiumguajava Linn. \\
\end{tabular} & $\begin{array}{c}24-25 \mu \mathrm{m}, \text { Ambsubtriangular; } 13- \\
\begin{array}{c}16 \times 26-28 \mu \mathrm{m}, \text { oblate; Radially } \\
\text { symmetrical }\end{array}\end{array}$ & $\begin{array}{c}\text { Tricolporate, syncolpate, } \\
\text { parasyncolpate, oralalongate }\end{array}$ & $\begin{array}{l}\text { Exine } 1.5 \mu \mathrm{m} \text { thick, tectate surface } \\
\text { granular to pailate }\end{array}$ \\
\hline 24 & Sonchusoleraceuslinn. & \begin{tabular}{|c|}
$39-44 \mu \mathrm{m}$, Amb more or less \\
hexagonal with rounded corners, \\
sides straight to slightly convex; \\
$37-47 \mathrm{x} 40-45 \mu \mathrm{m}$ oblate \\
spheroidal , Radially symmetrical
\end{tabular} & $\begin{array}{l}\text { Tricolporate, colpi faint due to heavy } \\
\text { sculpture, oralalongate }\end{array}$ & \begin{tabular}{|c|} 
Exineupto $12 \mu \mathrm{m}$ thick, tectate, sexine \\
much thicker than nexine at ridges, \\
surface echinolophate), spines of \\
different sizes, upto $3 \mu \mathrm{m}$ long, \\
fenestralluminaupto 21 , polygonal to \\
irregular, psilate, 6-8 prominent ridges \\
are soon along the equator which join \\
the equatorial lacuna of both the \\
hemispherrrres
\end{tabular} \\
\hline 258 & Sapindusemarginatusvahi. & $\begin{array}{c}24-26 \mu \mathrm{m}, \text { Amb triangular, sides } \\
\text { straight or even slightly concave; } \\
18-20 \times 26-29 \mu \mathrm{m} \text {, oblate } \\
\text { (occasionally suboblate); Radially } \\
\text { symmetrical }\end{array}$ & $\begin{array}{l}\text { Tricolporate, colpi narrowly elliptic } \\
\text { long, tips acute, oralOlongate }\end{array}$ & $\begin{array}{l}\text { Exine } 2 \mu \mathrm{m} \text { thick on mesocolpia, } 1-1.5 \\
\mu \mathrm{m} \text { thick near apertures, surface psilate }\end{array}$ \\
\hline 26 & $\begin{array}{l}\text { Rungiarepens(Linn.) } \\
\text { Nees. }\end{array}$ & $\begin{array}{c}40-44 \times 25-26 \mu \mathrm{m}, \text { oblong; } \\
\text { Bilaterally symmetrical }\end{array}$ & $\begin{array}{l}\text { Diporate, pores circular, } 2.5 \mu \mathrm{m} \text {, in } \\
\text { diam, margin of the pores densely besete } \\
\text { with small processes }\end{array}$ & $\begin{array}{c}\text { Exine } 3 \mu \mathrm{m} \text { thick at poles, } 4.6 \mu \mathrm{m} \text { at } \\
\text { distinct rounded to irregular areolae ( } 2- \\
4 \mu \mathrm{m} \text { ) linearly aligned in the vicinity of } \\
\text { apertures, rest of the wall } \\
\text { microreticulate }\end{array}$ \\
\hline 27 & $\begin{array}{l}\text { Syzygiumcumini (Linn.). } \\
\text { Skeels. } \\
\end{array}$ & \begin{tabular}{|}
$16-18 \mu \mathrm{m}$, Amb triangular to \\
rarely quadrangular, sides slightly \\
concave; $10.5-12 \times 17-20 \mu \mathrm{m}$, \\
oblate; Radially symmetrical
\end{tabular} & $\begin{array}{c}\text { Tricolporate, rarely tetra colporate, } \\
\text { syncolpate, parasyncolpate, } \\
\text { oralalongate }\end{array}$ & $\begin{array}{l}\text { Exine } 1.25 \mu \mathrm{m} \text { thick, tectae, surface } \\
\text { granular to smooth }\end{array}$ \\
\hline 28 & Terminalia sp. & $\begin{array}{c}19-22 \mu \mathrm{m}, \text { Amb spheroidal; } 21-24 \\
\text { x20-22 } \mu \mathrm{m} \text {, subprolate; Radially } \\
\text { symmetrical }\end{array}$ & $\begin{array}{c}\text { Tricolporate, colpi alternating with } \\
\text { pseudocolpicolpi linear, tips acute } \\
\text { pseudocolpi almost equal the size of } \\
\text { colpi, ora more of less circular }\end{array}$ & $\begin{array}{l}\text { Exine } 1.5 \mu \mathrm{m} \text { thick, tectae, surface } \\
\text { psilate to locally finely granular }\end{array}$ \\
\hline 29 & Tridaxprocumbens Linn. & $\begin{array}{c}\text { 31-38 } \mu \mathrm{m}, \text { Amb rounded } \\
\text { triangular to squarish; } 30-35 \mathrm{x} 32- \\
38 \mu \mathrm{m} \text {, oblate spheroidal; Radially } \\
\text { symmetrical }\end{array}$ & $\begin{array}{l}\text { Tri to tetra colporate, colpi linear, } \\
\text { sharply tapering, ora faint, circular }\end{array}$ & $\begin{array}{l}\text { Exine } 5 \mu \mathrm{m} \text { ( without spines) thick, } \\
\text { tectate, surface echinate, spines } 6 \mu \mathrm{m} \\
\text { long, } 2.5 \mu \mathrm{m} \text { in diam, at base }\end{array}$ \\
\hline
\end{tabular}

Volume 5 Issue 6, June 2016 www.ijsr.net

Licensed Under Creative Commons Attribution CC BY 


\section{International Journal of Science and Research (IJSR) \\ ISSN (Online): 2319-7064}

Index Copernicus Value (2013): 6.14 | Impact Factor (2015): 6.391

Table 3: Showing pollen morphology of Non-melliferous taxa

\begin{tabular}{|c|c|c|c|c|}
\hline Sr. No. & Pollen types & $\begin{array}{c}\text { Pollen Size, Shape and } \\
\text { Symmetry }\end{array}$ & Aperture pattern & $\begin{array}{c}\text { Pollen wall (Sporoderm) Structure and } \\
\text { sculpture }\end{array}$ \\
\hline 01 & $\begin{array}{c}\text { Sorghum } \\
\text { vulagare Pers. }\end{array}$ & $\begin{array}{c}51-55 \mu \mathrm{m} \text {, spheroidal; Radially } \\
\text { symmetrical }\end{array}$ & $\begin{array}{c}\text { Monoporate, pore circular provided with } \\
\text { annulus, pore diam with annulus } 4.1 \mu \mathrm{m} \\
\text { without annulus } 3.3 \mu \mathrm{m}\end{array}$ & $\begin{array}{c}\text { Exine 1 } \mu \mathrm{m} \text { thick, tectate, surface faintly } \\
\text { granular to almost psilate }\end{array}$ \\
\hline 02 & $\begin{array}{c}\text { Typhaangustata } \\
\text { Bory. et Chaub }\end{array}$ & $\begin{array}{c}28-35 \mu \mathrm{m} \text {, ellipsoidal, triangular } \\
\text { or spheroidal; Radially } \\
\text { symmetrical }\end{array}$ & $\begin{array}{c}\text { Monoporate pore more or less circular 4-5 } \\
\mu \mathrm{m} \text { in diam, margin wavy, pore } \\
\text { membrane densely granular }\end{array}$ & $\begin{array}{c}\text { Exine } 2.5 \mu \mathrm{m} \text { thick, subtectate, surface } \\
\text { reticulate in places retipilate, reticulum } \\
\text { homobrochate, lumina polygonal to circular, } \\
\text { psilate, murisimplibaculate }\end{array}$ \\
\hline
\end{tabular}

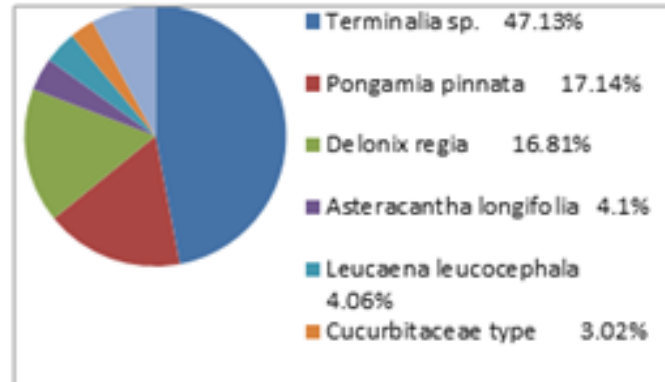

Figure 1.1: Palynograph of Nawargaon

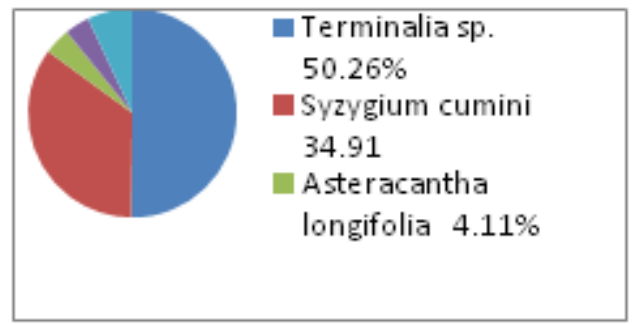

Figure 1.2:Palynograph of Delanwadi

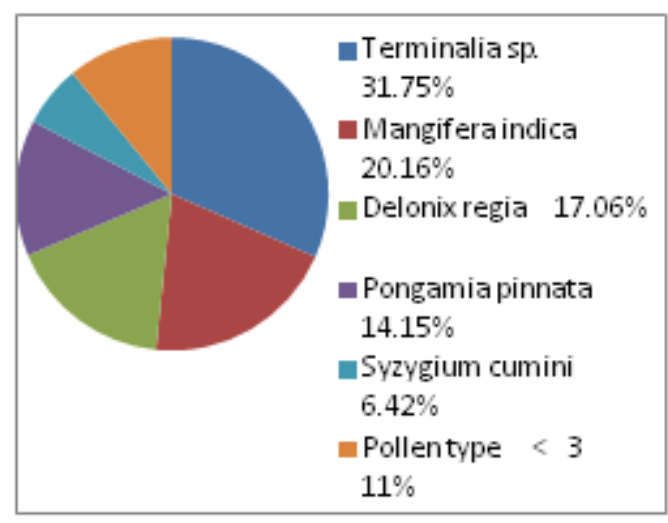

Figure 1.3: Palynograph of Kargata

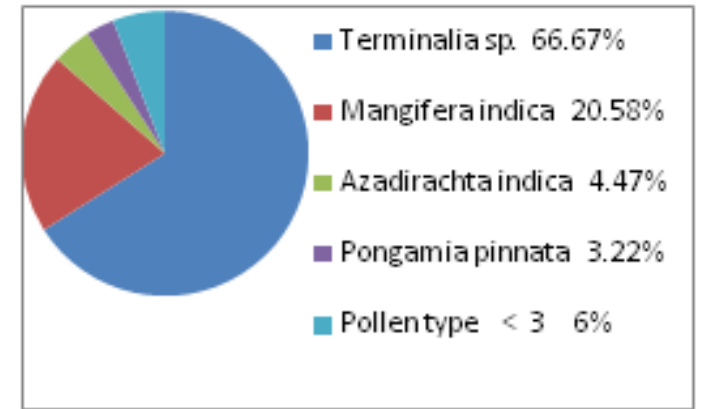

Figure 1.4: Palynograph of Wasera

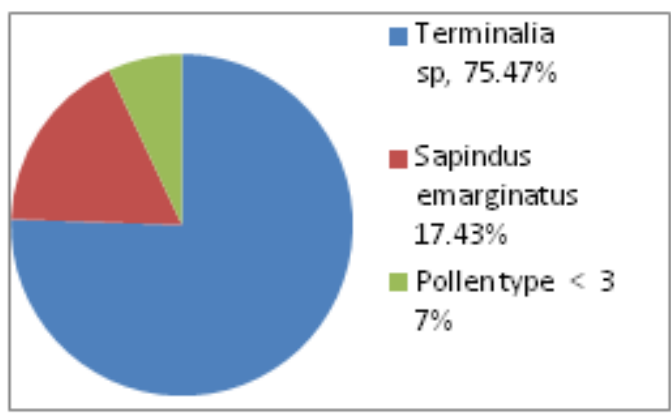

Figure 1.5: Palynograph of Shirkada

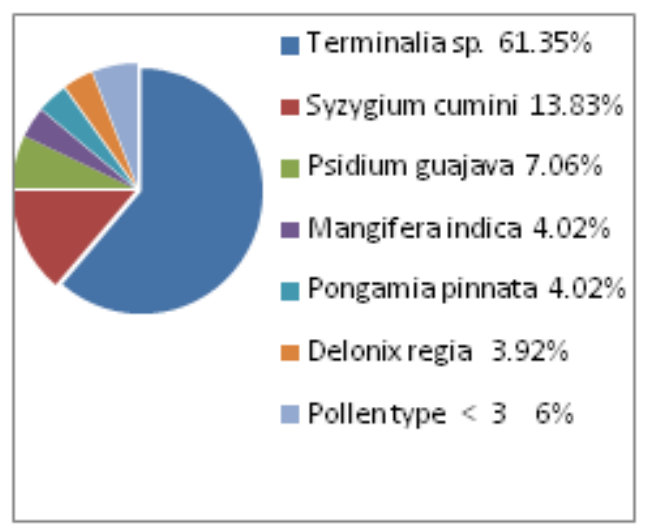

Figure 1.6: Palynograph of Gadbori

Volume 5 Issue 6, June 2016 www.ijsr.net 
International Journal of Science and Research (IJSR)

ISSN (Online): 2319-7064

Index Copernicus Value (2013): 6.14 | Impact Factor (2015): 6.391

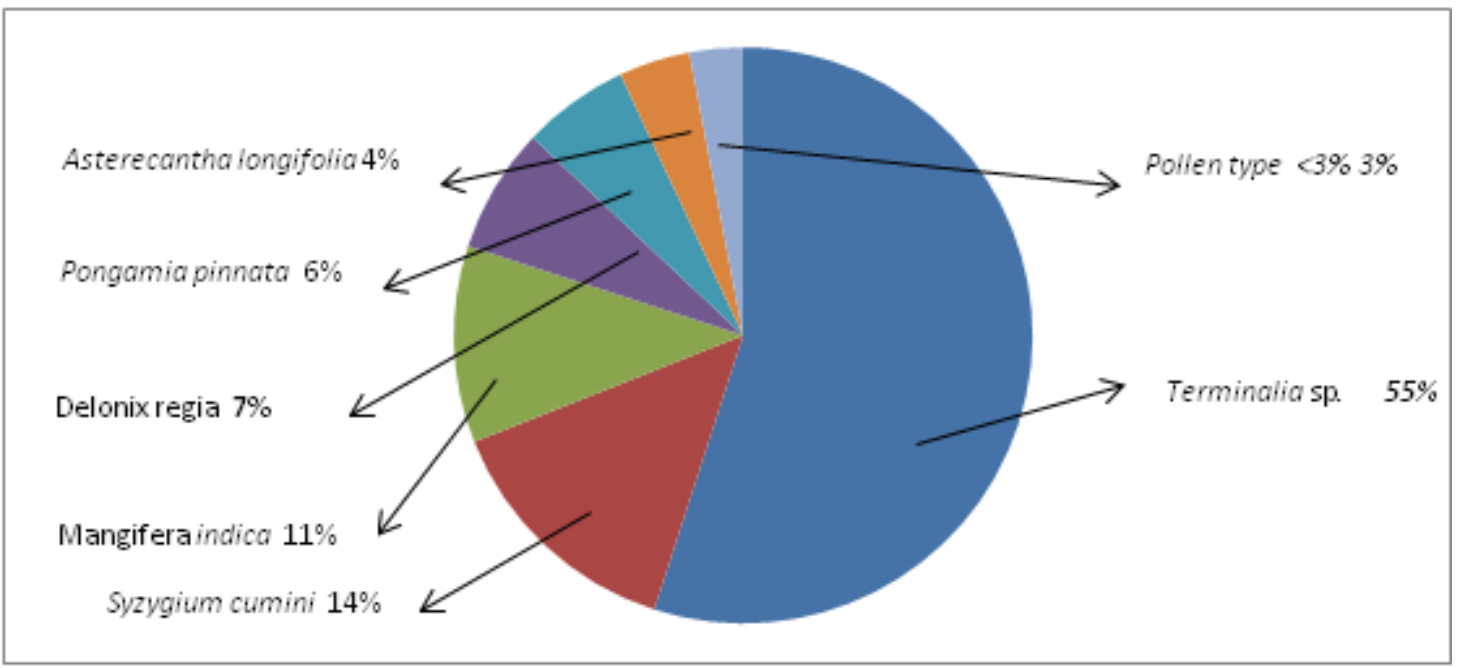

Figure 1.7: Composite palynograph of summer honeys from Sindewahi tahsil
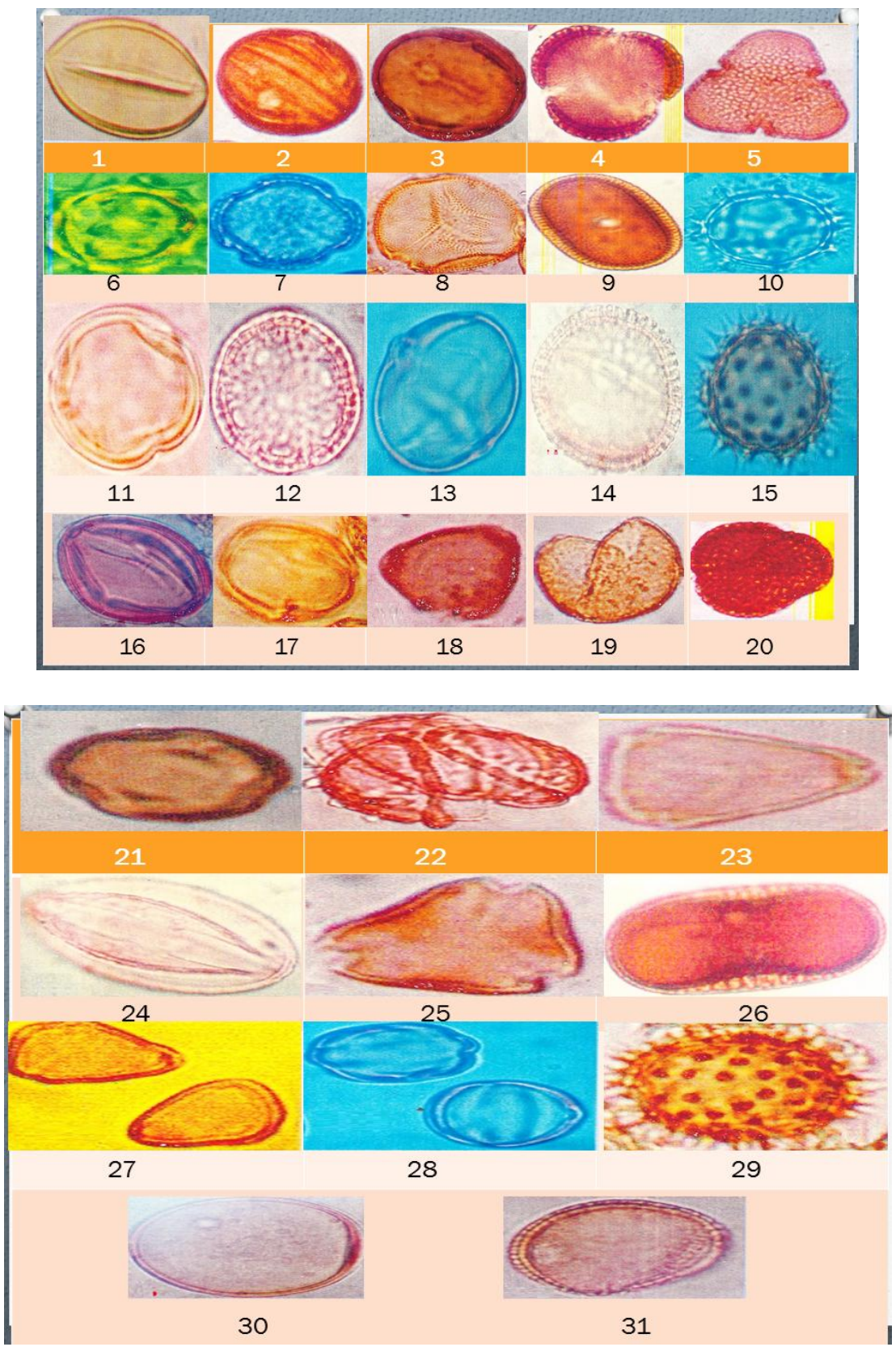

Figure 2: Microscopic photograph of pollen grains found in honey sample

Volume 5 Issue 6, June 2016 www.ijsr.net

Licensed Under Creative Commons Attribution CC BY 


\section{International Journal of Science and Research (IJSR) \\ ISSN (Online): 2319-7064}

Index Copernicus Value (2013): 6.14 | Impact Factor (2015): 6.391

1) Allium cepa 2)Asteracanthalongifolia

3) Azadirachtaindica 4) Brassicasp.

5) Bombaxceiba 6) Bidenspilosa

7) Citrussp. 8) Cucurbitaceae type

9) Rungiarepens 10) Sonchusoleraceus

11) Caseariaelliptica 12) Celosia argentea

13) Capsicumannuum 14) Delonixregia

15)Helianthusannuus 16) Leucaenaleucocephala

17) Mangiferaindica 18) Eucalyptus sp.

19) Clerodendrumsp. 20) Maytenusemarginata

21) Pongamiapinnata 22) Careyaarborea

23)Pisidiumguajava 24)Prosopisjuliflora

25) Sapindusemarginatus 26) Justiciaprocumbens

27) Syzygiumcumini 28) Terminalia sp.

29) Tridaxprocumbens 30) Sorghum vulgare

31) Typhaangustata

All together 31 pollen types ( 29 of melliferous and 2 of nonmelliferous taxa) referable to 23 families have been recorded from these samples (Photoplate). The sample (CHN-SINNaw) showed the maximum number of pollen type (13) and the sample (CHN-SIN-Was), the minimum number (8).

In the sample (CHN-SIN-Kag) however the pollen of Typhaangustata were found to be good number (11.58\%). The absolute pollen counts ranged from $161,000 / \mathrm{g}$ to 456 , 000/g and the HDE/P ratio ranged from 0.01 to 0.03 and represented by fungal spores (Table 1).

The details of the pollen analysis of the 6 honey samples (melliferous/non-melliferous) are represented in table 2 . Similarly individual palynograph (Pollen spectra) of each honey sample and composite palynograph was also given to show the pollen contents of the samples of Sindewahi tahsil. The distinguishing morphological features of the pollen types encountered in the present study are given below. The bee plants of Sindewahi tahsil are referable to 3 categories:

1) Crop plants:, Allium cepa, Brassica sp., Capsicum annuumand Helianthus annuusSorghum vulgare.

2) Arborescent taxa/shrub: Terminalia sp., Pongamiapinnata, Delonixregia, Syzygiumcumini, Rungiarepens, Eucalyptus sp., Prosopisjuliflora, Pisidiumguajava, Bombaxceiba, Cucurbitaceae type, Mangiferaindica, Caseariaelliptica, Sonchusoleraceu, Justicaprocumbens, Clerodendrum, Careyaarboreya, Mayeteneousemarginal, Leucaenaleucocephala, Sapindusemarginatus.

3) Herbaceous weeds: Asteracanthalongifolia, Tridaxprocumbens, Sapindusemarginatus, Celosia argentea, Bidenspilosa.Of these three categories the arborersent plants Terminalia sp. constitute the chief bee forage plants in this tahsil during summer season. Besides the other arborersent plants Delonixregia, Pongamiapinnata, Azadirachtaindicarepresents most preferred nectar sources for the honey bees. Our observation indicates that Terminalia $s p$. represent abundant nectar and pollen sources to Apisdorsata.

The region selected for the present study has good potential for sustaining bee keeping ventures because of the diversity of nectar and pollen taxa. Since Terminalia $s p$. are member of combretaceae is major sources of forage for honey bees therefore efforts should be made to increase it's cultivation. The other plant encountered in these honey samples are the member of families like Acanthaceace, Anacardeaceace, Mimoscae, Caesalpiniaceace, Celastraceace, Myrtaceace, Samydaceae, Menisphermaceace, Liliaceace, Capparidaceae, Amaranthaceae, Cleomaceae, Solanaceae, Papillionaceae and Sapindaceae in this area.

To improve the bee-keeping industry a proper understanding and mutualism between bees and available plant taxa in the region and in a particular season is necessary. The identified taxa were not only the economic crops but also play an important role in the development of bee-keeping in this region.

This data reflects the floral situation of the place where particular honey was produced and the identification of geographical origin based on the presence of a combination of pollen types of that particular area.

\section{Conclusion}

The present melittopalynological concepts based on 06 honey samples and Apisdorsata from seasons clearly brought to light that SINDEWAHI tahsils of Chandrapur district with large forest area in which Terminaliasp. with extensive plantations of Prosopisjuliflora, Delonixregiaand Leucaenaleucocephalanot only provide the bulk of giant bee honey, but also have the potential for attracting beekeeping industry involving colonies of the Indian Hive Bee, Apiscerena on modest commercial scale.

Adequate primary sources of nectar and pollen, augmented by secondary or alternate source when required for prolonged periods, availability unpolluted water resources and suitable soil types with enough moisture are prerequisites of paramount importance for successful operation for bee-keeping ventures in any area. A consideration of all these parameters clear by indicates that the forest area of Sindewahi, in particular merit serious attention for establishing bee-keeping enterprises for commercial honey production in Chandrapur district. Maintenance of apiaries in the vicinity of agricultural crops, in additional providing enough harvest of honey, also results in enhanced crop yield as a consequence of higher degree of pollination. Summer represents the honey flow season period with adequate sources of bee forage in the forest area with agricultural tracts; successful operation of apicultural activities necessitates migration of apiary colonies to nearby deciduous forest areas during summer months, for uninterrupted production of commercial honey.

\section{Future Scope}

The chief aim of this study is to exploit the methodology of melittopalynology for recognizing the major bee forage plants and make suitable recommendations and meaningful suggestions for promoting the cause of bee-keeping (apiary) industry and honey production in some specific areas of Chandrapur district. This would incidentally facilitate in realizing an additional source of income to all such farmers, who would take up beekeeping ventures on commercial scale. This will create interest in the present circumstances of non-employment.

\section{Volume 5 Issue 6, June 2016}




\section{References}

[1] Agashe SN, RangaswamyBE. Melittopalynological studies of honey samples from Bangardka, Dakshin Kannada district, Karnataka, Indian Bee J., 1997; 59(1), 8-11.

[2] Bhargava HR, Jyothi JVA, Bhushanam M, Surendra NS. pollen analysis of Apishoney, Karnataka, India. Apiacta, 2009; 44: 14-19.

[3] Bhusari NV, Mate DM, Makde KH. Pollen of Apishoney from Maharashtra, Grana., 2005; 44216-224.

[4] Erdtman G. The Acetolysis method. A revised description sv, Bot. Tidskr., 1960;54; 561-564.

[5] Kalpana TP, Ramanujam CGK. Melittopalynological investigation of honeys from Apisfloreaand Apisdorsatahives, Biovigyanam, 1991; 17(1):12-23.

[6] Kumar Rakesh, Jagtap SD. Melittopalynological studies on Rock bee honey samples during Winter season in Purander area of Pune district in Maharashtra. J. of Palynol., 1988; 34; 157-162.

[7] Louveaux J, Maurizio A, Vorwhol G. Methods of Melissopalynology, Bee World, 1978; 59; 139-157

[8] Moses TS, Singh PR, Joshi A, Suryanarayana MC. Evaluation of sources of pollen to honey Bees at Vijayarai (Andra Pradesh), proc. 5th All India Symp. Palynol, October 7-9, 1987, Dept. Bot., Inst. Sci, Nagpur, India, 1987, pp. 65-71.

[9] Nair PKK. A pollen analytical study of Indian honeys. J. Ind. Bot. Soc., 1964, 43:179-191.

[10] Phadke RP. Physico chemical composition of major unifloral honeys from Mahabaleshwar (Western Ghats). Ind. Bee J.1962; 24 59-65.

[11] Ramanujam CGK, Khatija F. Melittopalynogy of the agricultural tracts in Guntur district, Andhra Pradesh. $J$. Indian Inst. Sci., 1991, 71, 25-34.

\section{Author Profile}

Dr. Laxmikant N. Borkar, M.Sc., M.Phil., Ph.D. Head \& Assistant Professor, Department Of Botany, S.S.Jaiswal Arts, Comm and Science College, Arjuni Morgaon. Dist. Gondia 441701. He has Teaching Experience of 7 years. No of Research paper published 12

Dr. Devendra M. Mate, M.Sc., Ph.D. Head \& Associate Professor, Department Of Botany, Nutan Adarsh Arts, Comm, and smt M.H. Wegad Science College, Umrer. Dist.Nagpur441203. He has Teaching Experience 27 years. No of Research paper published 14. Research Guidance Ph.D. Awarded 01, Ph.D. Thesis Submitted 01, Four students persue Ph.D. Degree Ex. Chairman of board of studies RTM Nagpur University Nagpur 